\title{
Solution of The Combined Environmental Economic Dispatch Problem Using Multi-Objective Cat Swarm Optimization
}

\author{
Sarat Kumar Mishra and Sudhansu Kumar Mishra \\ Department of Electrical and Electronics Engineering \\ Birla Institute of Technology \\ Mesra (Ranchi), India \\ mishra.sarat@gmail.com
}

\begin{abstract}
This paper deals with the realistic combined environmental economic dispatch (CEED) problem, considering the running fuel cost along with emission as objectives taking power balance and operating limits of the generators as constraints. A new dynamic algorithm for multiobjective optimization, namely the modified multi-objective cat swarm optimization (MOCSO) has been formulated and successfully implemented to address the nonlinear multi-modal CEED problem. A new constraint handling technique is incorporated in the algorithm to satisfy the nonlinear constraints. Further, to select a single solution from the set of Pareto solutions, best tradeoff solution is obtained through fuzzy inference. A thorough investigation has been carried out on two standard test cases namely, the IEEE 14 bus and IEEE 30 bus test systems. The effectiveness of the algorithm is compared with other competitive evolutionary algorithms, such as the non-dominated sorting genetic algorithm (NSGA-II), strength Pareto evolutionary algorithm2 (SPEA2), multi-objective particle swarm optimization (MOPSO) and multi-objective differential evolution (MODE). Performance estimation is done on the basis of computational time, Pareto fronts and non-parametric performance measures. The statistical analysis is also performed, to show the superiority of the proposed modified MOCSO algorithm. Examination of the performance measures shows that the modified MOCSO provides good Pareto solutions while maintaining diversity. It provides a wide scope to trade a balance between running cost and environmental emission of thermal power plants under different types of challenging constraints.
\end{abstract}

Keywords: Cat swarm optimization, combined environmental economic dispatch, multiobjective optimization, non-dominated sorting

\section{Introduction}

The economic load dispatch serves as the basic tool for generation scheduling of a multi-unit power system. The schedule for the most economic operating condition of the units committed is found for a particular load demand. Due to the bad effect on the environment, emission from the thermal power plants needs to be minimized along with the running cost. The plant emission and running cost incurred in the unit rise with the quantity of power generated. These two objectives vary nonlinearly as functions of unit power generation. Total running cost is not necessarily minimum when the total emission goes minimum. The converse is also not true indicating that it is an inherently multi-objective problem. Each committed unit must generate power within its own minimum and maximum feasible limits; these limits can be taken as the first set of constraints. Further, the total power generated from all the units must just cater to the total load demand plus system transmission losses. This condition of power balance acts as another constraint. Improvement in the quality of fuel leads to lower emission but at a higher cost. Hence, achieving the two objectives, low cost and low emission is not possible simultaneously. Thus, the combined economic emission dispatch problem is multimodal and multi-objective in nature and is associated with nonlinear constraints.

Many derivative based methods are used to solve this nonlinear scheduling problem; these include the Gauss-Siedel, Newton-Raphson and Lagrangian multiplier methods [1-2]. These methods suffer from drawbacks of local optimum values and do not yield global solutions. The

Received: March $5^{\text {th }}, 2019$. Accepted: May $20^{\text {th }}, 2021$

DOI: $10.15676 /$ ijeei.2020.13.2.2


CEED problem being inherently multi-objective in nature requires tradeoff between the objectives. Initially, authors used to convert the problem into a single objective one by weighted sum approach [3]. They assigned different weightage to the two objectives and carried out optimization for graded variation of the weightage. All these optimal points are represented on a graphical front arranged on ascending values of the objectives. Such a graphical front carrying tradeoff relations of many non-commensurable objectives is termed as a Pareto optimal front. The Pareto front obtained through the weighted sum approach is not uniform and the tradeoff information is often lost for the concave objective functions. Moreover, they require the same algorithm to run many times with different weightage values to obtain a single Pareto front. Many researchers adopted heuristic approaches to overcome these drawbacks [4-5]. The evolutionary algorithms consider the two objectives simultaneously and draw a tradeoff curve with one algorithm run. These are termed as evolutionary multi-objective optimization algorithms and have better performance over the previous weighted sum algorithms. Chiang et al. [6] suggested an improved version of genetic algorithm using the $\epsilon$-constraint technique along with a multiplier updater and achieved a better speed of convergence and improved exploration capability.

The non-domination sorting genetic algorithm-II (NSGA-II) proposed by Deb et al. [7-8] introduced Pareto ranking and assignment of crowding distance for striking a tradeoff between the objectives. A single run of the algorithm draws the Pareto front for the non-commensurable conflicting objectives. But it suffers due to the dependence on the complicated biological crossover and mutation operators. A modification to the NSGA-II made by Brar et al. [9] incorporated the fuzzy decision system and demonstrated better search capability. Further refinement was introduced by Muthuswamy et al. [10] by modifying the crowding distance to a dynamic reference frame. This modification could result in improvement in the diversity of the Pareto solutions. However, all these approaches do not perform well for discontinuous objective functions. Therefore, a new algorithm called artificial bee colony (ABC) optimization has been implemented by Panigrahi et al. [11] which resulted in better reliability and higher rate of convergence for objective functions with nonlinear ramp rate limits and prohibited operating points. This has been further improved to the improved artificial bee colony (IABC) by incorporation of chaos ques skill in the search process. Another population based algorithm called Particle swarm optimization (PSO) inspired by the behavioral pattern of swarms has been employed by Mori et al. [13]. The authors formulated adaptive parameters in the conventional PSO and demonstrated improved results. Hadji et al. further refined the PSO algorithm through a time varying acceleration constant in PSO to achieve better exploration capability [14]. A new algorithm named the differential evolution (DE) came up recently which uses the difference of two individuals to evolve a new individual from a third one [15]. It has less dependence on biological operators and results in better global optimization. Meza et al. introduced a new concept of spherical pruning to further improve its exploration capability [16]. The algorithm has been further modified by Di et al. for better constraint handling ability with the introduction of an operator called marginal analysis correction factor [17].

Kuo et al. [18] proposed an integrated approach to solve this bi-objective problem for a realistic power system of Taiwan. They applied the simulated annealing technique and developed a correlation between the two objectives to find the best compromise. Arriagada et al. [19] solved the problem for a power system integrated with renewable sources and applied a probabilistic model. They considered the uncertainties, short term control and other factors incorporating Monte Carlo solution strategy. These papers have not taken the Pareto front approach which provides a good flexibility to choose a compromise between the two objectives as per the affordability and other issues. Elsakaan et al. [20] formulated a framework for scheduling of power in micro grids basing on the CEED problem through an enhanced moth flame optimization algorithm. Dong and Wang [21] used a weighted sum approach incorporating the Kernel tricks to solve the multi-objective problem for a number of real time power systems with valve point loading. An improved indicator and crowding based optimization algorithm has been proposed by Sun et al. [22] to solve the CEED problem with valve point loading for different test cases. 
In this paper, we have implemented a new constraint handling mechanism and suitably incorporated it in the multi-objective optimization (MOP) algorithms. Finally, the effectiveness of the proposed algorithm is tested by considering some of the standard conditions. Also, a fuzzy decision-making system has been implemented to arrive at the best trade-off point from the set of non-dominated solutions.

\section{Review of Multi-Objective Optimization}

A multi-objective optimization algorithm solves optimization problems with many objectives that are conflicting in nature. The solution process starts with a population of individuals each one of which represents a solution having many decision variables. The MOP is assigned with a number of conflicting objective functions as per the problem statement. The MOP arrives at the final optimum solution through tradeoff between the objective functions taking the constraints into account. In the multi-objective CEED problem we consider the minimization problem that can be generalized as:

$$
\text { Minimize } f(\vec{x})=\left(f_{1}(\vec{x}), f_{2}(\vec{x}), \ldots, f_{M}(\vec{x})\right)
$$

Subject to constraints:

$$
\begin{aligned}
& g_{j}(\vec{x}) \leq 0 ; \quad j=1,2, \ldots, J \\
& h_{k}(\vec{x})=0 ; \quad k=1,2, \ldots, K
\end{aligned}
$$

where, $\vec{x}$ is a $\mathrm{N}$ dimensional vector represented as $\vec{x}=\left[x_{1}, x_{2}, \ldots, x_{N}\right]^{T}$

The boundary restrictions on the search space may be represented by lower and upper bounds as

$$
l b_{i} \leq x_{i} \leq u b_{i} ; \quad i=1,2, \ldots, N
$$

A solution vector $\vec{u}=\left[u_{1}, u_{2}, \ldots, u_{N}\right]^{T}$ dominates over another solution $\vec{v}=\left[v_{1}, v_{2}, \ldots, v_{N}\right]^{T}$ if and only if

$$
\left.\begin{array}{rl}
f_{i}(\vec{u}) & \leq f_{i}(\vec{v}) \quad \forall i \in[1,2, \ldots, M] \\
f_{i}(\vec{u}) & <f_{i}(\vec{v}) \text { for at least one } i \in[1,2, \ldots, M]
\end{array}\right\}
$$

The non-inferior solutions which cannot be dominated by any other solution in the feasible zone of search space are called as non-dominated solutions. The curve that shows the mapping of the objective function values of such non-dominated points is referred to as the Pareto optimal front (POF).

$$
P O F=f(\vec{x})=\left[\left\{f_{1}(\vec{x}), f_{2}(\vec{x}), \ldots, f_{k}(\vec{x})\right\} \mid \vec{x} \in p\right]
$$

where $\mathrm{p}$ is the set of non-inferior individuals in the population.

\section{Combined Environmental Economic Dispatch (CEED) Problem}

The economic load dispatch leads to an operating schedule of generators such that the fuel cost involved is minimum. The committed generating units combined together supply the total demanded power along with the losses incurred due to transmission while obeying the prescribed lower and upper bounds. The fuel cost for each unit depends on the real power generated, turbinegenerator efficiency and the fuel quality. The approximate fuel cost characteristics for each generating unit is a polynomial of the corresponding generated power of the second order.

Thus, for the $i$ th generator supplying a real power $P_{G_{i}}$, the fuel cost is given by

$$
F_{i}=a_{i} P_{G i}^{2}+b_{i} P_{G i}+c_{i}
$$

where, $a_{i}, b_{i}$ and $c_{i}$ are cost coefficients.

The $i$ th generator causes an approximate emission of

$$
E_{i}=\alpha_{i}+\beta_{i} P_{G i}+\gamma_{i} P_{G i}^{2}
$$

where, $\alpha_{i}, \beta_{i}, \gamma_{i}$ are emission coefficients.

A minimum value of total fuel cost is the outcome of the ELD program that decides optimum generating schedule given by

$$
F_{T}=\sum_{i=1}^{N} F_{i}
$$

subject to the constraints.

The power balance criterion states that the total generation of real power must be the same as the power demanded plus losses incurred during transmission

$$
\sum_{i=1}^{N} P_{G i}=P_{D}+P_{L}
$$


where $P_{D}$ is the total demanded load and $P_{L}$ is the loss incurred in transmission. It is represented by Kron's equation

$$
P_{L}=\sum_{i=1}^{N} \sum_{j=1}^{N} P_{G i} B_{i j} P_{G j}+\sum_{i=1}^{N} B_{i 0} P_{G i}+B_{00}
$$

The constants $B_{i j}, B_{i 0}$ and $B_{00}$ are dependent on the line parameters. The real power generation from the units should obey the prescribed lower and upper limits.

$$
P_{G i(\min )} \leq P_{G i} \leq P_{G i(\max )} ; i=1,2, \ldots, N
$$

The CEED problem is required to minimize both the emission and fuel cost simultaneously. The emission and fuel cost functions are conflicting in nature as their coefficients are different. Thus, the CEED problem is inherently a multimodal multi-objective optimization problem which does not have a unique solution. The solution for such problem is obtained through tradeoff between the objectives and usually represented in the form of Pareto optimal front.

The statement of the problem can be expressed as:

$\underset{P_{G}}{\operatorname{minimize}}\left[F_{T}\left(P_{G}\right), E_{T}\left(P_{G}\right)\right]$

Subject to: $g\left(P_{G}\right)=0$

$$
h\left(P_{G}\right) \leq 0
$$

where the equality constraint is given by equation (14) and inequality constraint by equation (15) below.

$$
\begin{aligned}
& \sum_{i=1}^{N} P_{G i}-P_{D}-P_{L}=0 \\
& P_{G i}-P_{G i}^{\max } \leq 0 ; P_{G i}^{\min }-P_{G i} \leq 0
\end{aligned}
$$

\section{A. Non-Dominated Sorting Genetic Algorithm-II (NSGA-II)}

The NSGA-II algorithm has been originally framed by Deb et al. to deal with the solution of multi-objective optimization problems [7-8]. This algorithm is a population based one that starts with a randomly selected population obeying the lower and upper limits. Its principle depends mainly on three operations: rank assignment according to density, estimation of crowding distance and non-dominated sorting. According to rank and crowding distance, the solutions are classified into different layers. The solutions with lower crowding distance are rejected in order to maintain those with high value of diversity. Also to ensure good quality of solutions, the individuals with lower ranks are rejected. This algorithm has a merit of lower computational complexity and maintenance of elitism.

\section{B. Strength Pareto Evolutionary Algorithm2 (SPEA2)}

Formulated by Zitzler and Thiele in 1999, this algorithm uses the operator binary tournament for selection of mating pool on the basis of fitness measure [23]. Dominance rank and dominance count is assigned to each individual for ascertaining its non-dominance over other individuals. The fitness value of the individual signifies the number of individuals dominated by it and those who dominate it. An archive is formed containing the non-dominated individuals and the current pool. In case the archive size is less than the predetermined number of individuals, then it is compensated with dominated individuals from the current pool, else few individuals are deleted using a truncation operator. The truncation operator uses the distance of the individual from its closest neighbor.

\section{Multi-objective Particle Swarm Optimization (MOPSO)}

A population based algorithm inspired by the group behavior of birds as proposed by Eberhart and Kennedy [24] has been popularly termed as the Particle swarm optimization (PSO). The flock of birds called as a swarm has a typical movement pattern to maximize the chance of getting food. The members of the flock follow the individual with the best position (gbest) and simultaneously each individual follows its own best position (pbest). Thus, the algorithm performs both local and global search optimization. Initially, each individual is assigned a position and velocity within the search space randomly. The fitness of the individuals is 
evaluated in terms of the objective functions and the global best individual is identified. The $i$ th individual with the best position (pbest) is given by $p_{i}$, that has the best fitness value.

where, $p_{i}=\left(p_{i 1}, p_{i 2}, \ldots, p_{i N}\right)$

The velocity of the individual is updated from the old value given by eq (17) to the new velocity as shown in eq (18) respectively.

$$
\begin{aligned}
& V_{i}=\left(v_{i 1}, v_{i 2}, \ldots, v_{i N}\right) \\
& v_{i d}(t)=w v_{i d}(t-1)+c_{1} r_{1}\left(p_{i d}-x_{i d}\right)(t-1)+c_{2} r_{2}\left(p_{i d}-x_{i d}\right)(t-1)
\end{aligned}
$$

and the position of each particle is then updated as

$$
x_{i d}(t)=x_{i d}(t-1)+\chi v_{i d}(t)
$$

where, $d=1,2, \ldots, D$ is the number of dimensions of the variables for the particle $i=1,2, \ldots, N$. $\chi$ is the constriction factor that restricts the magnitude of velocity. $w, c_{1}$ and $c_{2}$ are weight parameters and $r_{1}, r_{2}$ are constants of acceleration denoted by random numbers in the range [0, 1]. This method of improvement of position and velocity is applied to the non-dominating vectors to solve the multi-objective problem [14], [25] \& [26].

\section{Multi-objective Differential Evolution (MODE)}

Stern and Price framed a new algorithm named as differential evolution (DE) that evolves a new population which is created as a result of normalization of the existing vectors by addition of difference between others from the current population [15]. The algorithm has been modified by Meza et al. [16] to solve problems with a number of conflicting objectives and subsequently termed as multi-objective optimization using differential evolution (MODE). The MODE algorithm has been shown to improve the diversity and quality of Pareto solutions. The two main operators used by this algorithm are crossover and mutation. It starts with a random set of individuals in the feasible search area and updates the population set in subsequent iterations. A differential vector is formed from two different individuals other than that undergoing mutation; this differential vector is added to the target with a scaling factor as

$$
v_{i, g}=x_{r 0, g}+F \cdot\left(x_{r 1, g}-x_{r 2, g}\right)
$$

Where $v_{i, g}$ is the mutant vector generated from the target vector $x_{r 0, g}$ and $x_{r 1, g}, x_{r 2, g}$ are two other vectors $F \in(0,1+)$ is the scale factor controlling the rate of evolution.

A new trial vector (child) is further generated from the mutant vector and the target through crossover.

$$
u_{i, g}=u_{j, i, g}=\left\{\begin{array}{lr}
v_{j, i, g} \text { if }\left(\operatorname{rand}_{j}(0,1) \leq C_{r} \text { or } j=j_{\text {rand }}\right) \\
x_{j, i, g} \text { otherwise }
\end{array}\right.
$$

Where the probability of crossover $C_{r} \in[0,1]$

The fitness of the parent and child are evaluated to select the best one for the next iteration.

$$
x_{i, g+1}= \begin{cases}u_{i, g} \text { if } f\left(u_{i, g}\right) \leq f\left(x_{i, g}\right) \\ x_{i, g} & \text { otherwise }\end{cases}
$$

\section{E. Multi-objective Cat Swarm Optimization (MOCSO)}

This optimization algorithm was proposed by Chu and Tsai in 2006. It mimics the social behavior of animals belonging to the cat family. Cats spend much of their time to rest, but they are very alert and chase the moving preys in a very small time. The hunting pattern of cats is classified into two modes: 'Seeking mode' and 'Tracing mode'.

In this algorithm, a random set of the population in the $\mathrm{D}$-dimensional search space is created. Each cat representing a probable solution is associated with a position, velocity and a fitness value. Normally, a small number of cats chase the prey (tracing mode) while all others are at rest keeping an eye on the moving preys (seeking mode). A mixture ratio (MR) of small value is set 
which defines the ratio of the numbers of cats in tracing mode to those in seeking mode. The optimization process is carried out by the combined action of the entire population [27].

The steps followed are as follows:

a. Initialize the population of $\mathrm{N}$ cats in the $\mathrm{D}$ dimensional search space considering lower and upper limits, initialize position and velocity of each cat.

b. Set MR, constraints, fitness criteria and convergence rule.

c. Create seeking memory pool from the population in accordance with MR, mark rest as tracing mode.

d. Evaluate the position of each cat for constraint and Pareto dominance; store the nondominated ones in one archive.

e. Apply the process of seeking mode to cats in seeking mode pool and tracing mode to rest of the cats.

f. Check for termination and convergence criteria; if not satisfied go to next iteration.

\section{Seeking mode}

This corresponds to the global search and is applied to the pool of cats in seeking mode. The steps for this mode are:

a. Apply mutation to randomly selected cats and evaluate their fitness.

b. Update the archive with the non-dominated position of cats.

\section{Tracing mode}

It is the same as the local search process. The steps involved are:

a. Evaluate the fitness of the cats under tracing mode and identify the one with the best position among them. Mark the position of the local best as $x_{g}=\left(x_{g 1}, x_{g 2}, \ldots, x_{g D}\right)$

b. Update the velocity of all the cats using

$v_{i d}=w \times v_{i d}+c \times r \times\left(x_{g d}-x_{i d}\right)$; for $i=1,2, \ldots, N$ and $d=1,2, \ldots, D$

where $w$ is the inertia weight, $c$ is the constant of acceleration, $r$ is a random number $\in[0,1]$

c. Update the position of all the cats using $x_{i d}=x_{i d}+v_{i d}$; for $i=1,2, \ldots, N$ and $d=$ $1,2, \ldots, D$

d. If the position of a cat goes out of the feasible area of the search space, then its direction of movement is reversed by multiplying its velocity with $(-1)$.

e. Evaluate the fitness of each cat.

f. Update the archive with the non-dominated positions of these cats.

We propose a modified multi-objective cat swarm optimization (MMOCSO) algorithm which takes care of the two objectives, namely the fuel cost and emission with the nonlinear power balance condition and generator limits as constraints.

\section{Implementation of CEED Problem using MMOCSO algorithm}

In this paper, we have implemented a modification in the constraint handling and selection of the best trade-off point from the Pareto front. It has been formulated as follows.

\section{A. Constraint Handling}

While updating the population in any evolutionary algorithm, each new individual formed must satisfy the constraints specified in eq (10) -(15). The most important constraint being the power balance criteria which states that the total generated power must cater to the demanded load along with the transmission loss. This is because the transmission loss is a polynomial of the individual generations of the units. The new individual created from the algorithm may not satisfy the constraints. Thus, it becomes difficult to converge unless a continuous effort is made 
to contain the new individuals away from the infeasible area. Here, a recursive distributed constraint handling technique is proposed with the following pseudo codes:

The mismatch of power is termed as constraint $c$ is defined as

$$
\begin{gathered}
c=\sum_{i=1}^{N} P_{G i}-P_{D}-P_{L} \\
\left\{\begin{array}{c}
\text { if } c \leq \varepsilon \\
\text { then } P_{G i}=P_{G i} \\
\text { else } P_{G i}=P_{G i}-\frac{|c|}{N}
\end{array}\right.
\end{gathered}
$$

subject to $P_{G i}^{\min } \leq P_{G i} \leq P_{G i}^{\max }$

here, $\varepsilon$ is the maximum constraint violation allowed. The mismatch is evaluated and distributed over the $\mathrm{N}$ variables and the variables will go on updating until they satisfy the constraints. This process is repeated until all the points in the new population is brought into the feasible area of search space.

\section{B. Best Tradeoff Solution}

The search space explored by the algorithm locates various optimized points for different weights of the decision variables. A decision has to be made about the importance of each objective. A weight $w_{i}$ is assigned to the $i$ th objective function such that $\sum_{i=1}^{N_{O F}} w_{i}=1$; where $N_{O F}=$ No of objective functions. Each objective function is fuzzified by a membership function $\mu_{i}^{k}$ for every non-dominated solution $k$.

$$
\mu_{i}^{k}=\left\{\begin{array}{lr}
1 & O F_{i}^{k} \leq O F_{i}^{\text {min }} \\
\frac{O F_{i}^{\text {max }}-O F_{i}^{k}}{O F_{i}^{\text {max }}-O F_{i}^{\text {min }}} O F_{i}^{\text {min }}<O F_{i}^{k}<O F_{i}^{\text {max }} \\
0 & O F_{i}^{\text {max }} \leq O F_{i}^{k}
\end{array}\right.
$$

The overall normalized weighted membership value $\mu^{k}$ is estimated from; the solution point with maximum $\mu^{k}$ represents the best trade-off solution.

$$
\mu^{k}=\frac{\sum_{i=1}^{N_{O F}} w_{i} \cdot \mu_{i}^{k}}{\sum_{k=1}^{N_{S}} \Sigma_{i=1}^{N_{O F}} w_{i} \cdot \mu_{i}^{k}}
$$

\section{Performance Measures}

The quality of Pareto solutions obtained using different algorithms are normally assessed through various non-parametric performance metrics. In this work, four such metrics have been used to investigate the performance of the algorithms applied.

\section{A. Spacing (S)}

Spacing metric measures, the spread of the Pareto solutions. It is defined as

$$
\begin{aligned}
& S \triangleq \sqrt{\frac{1}{n-1} \sum_{i=1}^{n}\left(\bar{d}-d_{i}\right)^{2}} \\
& \text { where, } d_{i}=\min _{j}\left(\left|f_{1}^{i}(\vec{x})-f_{1}^{j}(\vec{x})\right|+\left|f_{2}^{i}(\vec{x})-f_{2}^{j}(\vec{x})\right|\right) \text { and } i, j=1,2, \ldots n
\end{aligned}
$$

$\bar{d}=$ mean of all $d_{i}$ and $n$ is the number of non-dominated solution vectors found so far. A lower value of $S$ indicates a better Pareto solution.

\section{B. Diversity Metric ( $\Delta$ )}

The diversity metric estimates how evenly the non-dominated solutions are distributed in the feasible search space. It is estimated by calculating the Euclidean distances as follows

$$
\Delta=\frac{d_{f}+d_{l}+\sum_{i=1}^{N-1}\left|d_{i}-\bar{d}\right|}{d_{f}+d_{l}+(n-1) \bar{d}}
$$


where, the distance between the consecutive solutions in the set of non-dominated solutions is denoted by $d_{i}$. The mean of these distances is $\bar{d}$. The first and last Euclidean distance are represented by $d_{f}$ and $d_{l}$ respectively. The better the diversity, the lower value of $\Delta$ metric; $\Delta=$ 0 means the non-dominated solution vectors are uniformly distributed on the Pareto front.

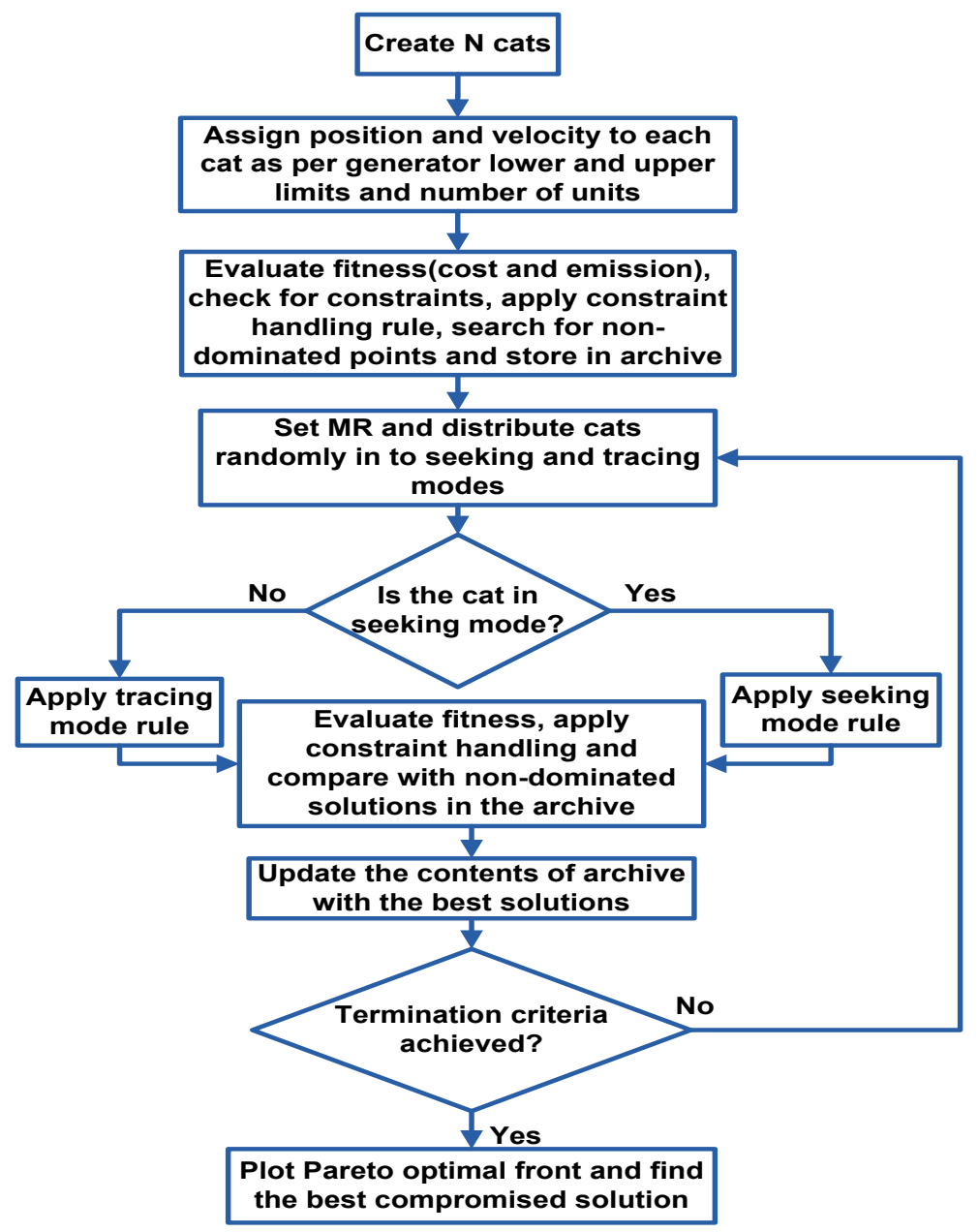

Figure 1. Flowchart for implementation of CEED problem using MMOCSO algorithm

C. Spread Diversity (SD)

This performance index helps in estimating the diversity between the solution sets at the extreme ends of the Pareto optimal front. A larger value of $S D$ indicates better performance.

$$
S D=\sqrt{\sum_{i=1}^{N_{O F}}\left(O F_{i}^{\max }-O F_{i}^{\min }\right)^{2}}
$$

$O F_{i}^{\min }$ and $O F_{i}^{\max }$ are the minimum and maximum values of the $i$ th objective function.

\section{Minimum Ideal Distance (MID)}

It indicates how evenly the non-dominated solutions are dispersed from ideality (i.e., minimum of the objective functions) in the search space. A lower value of $M I D$ is desirable for better performance [29].

$$
M I D=\sum_{j=1}^{N_{S}}\left(C_{j} / N_{S}\right)
$$


where

$$
C_{j}=\sqrt{\sum_{i=1}^{N_{O F}}\left(O F_{i}^{j}-O F_{i}^{\min }\right)^{2}}
$$

$O F_{i}^{j}$ is the value of the $i$ th objective function for the $j$ th non-dominated solution.

\section{Simulation Results}

The algorithms for CEED problem has been run for two standard test cases i.e., IEEE 14 bus and IEEE 30 bus. The former test case has five different generating units supplying a total load of $259 \mathrm{MW}$, whereas the latter consists of six units that deliver 283.4 MW of total load. All the generating units are committed during the study. The system data of these two test cases were obtained from the website www.ee.washington.edu/research/pstca. The coefficients for cost function and emission function were borrowed from other standard sources [30] as presented in the Appendix. The B coefficients found and used are also shown there. The solutions obtained using the algorithms NSGA-II, SPEA2, MOPSO, MODE, and MMOCSO were compared. The system used had a core i3 processor having $4 \mathrm{~GB}$ of RAM and $1.3 \mathrm{GHz}$ clock speed running on Microsoft Windows 10 in MATLAB environment.

For proper tuning of the parameters, the algorithms have been run several times with different parameters and the parameter values that yield the best results are finally used. A maximum of 300 iterations with a population size of 100 is chosen. The intermediate cross over ratio set as 1.2; Gaussian mutation with scale 0.1 and shrink 0.5 selected for NSGA-II. A scaling factor of 0.5 and the rate of crossover of 0.5 set for MODE with 100 populations. A position weight of 1 and velocity weight of 0.4 taken for MMOCSO with a population of 100 and a maximum 100 iterations.

The proposed algorithm is applied to the standard IEEE 14 bus and IEEE 30 bus test cases at rated load condition. The results have been compared with those of the other competing algorithms under the same load condition. The Pareto optimal fronts are shown in figure 2 and figure 3 It shows a clear advantage in favor of the MMOCSO. The generation schedule, computational time, the total operating fuel cost and total emission corresponding to best tradeoff solution obtained using the fuzzy decision-making system are shown in Table 1 and Table 2 for the IEEE 14 bus and IEEE 30 bus systems respectively. The proposed algorithm outperforms the others in terms of computational time. Further, the results show a lower fuel cost and the transmission loss involved in the system is low. Thus the proposed algorithm yields better solutions and satisfies the constraints with lower violations.

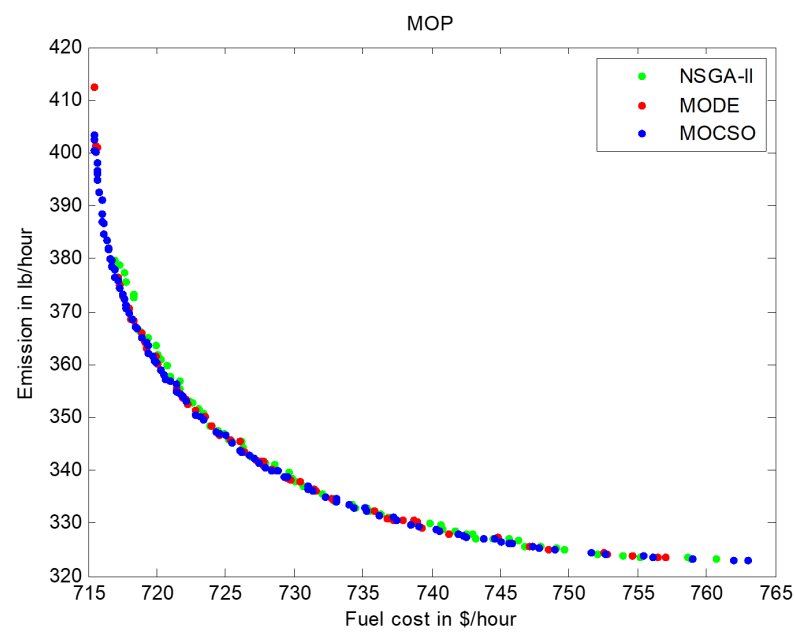

Figure 2. Comparison of Pareto fronts obtained for IEEE 14 bus system 


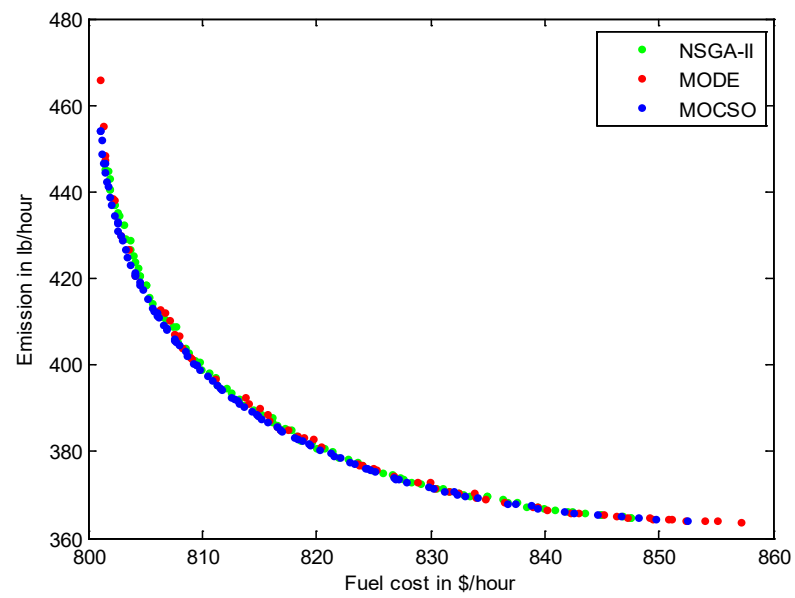

Figure 3. Comparison of Pareto fronts obtained for IEEE 30 bus system

The above Pareto optimal fronts represent the non-dominated solutions obtained from the three algorithms mentioned. The best tradeoff points are found out using the approach described previously in section 4 for equal importance to the objectives cost and emission. However, the importance to these objectives may be changed depending on the affordability issues. The best tradeoff values obtained from the different algorithms are mentioned in tables $1 \& 2$ for the IEEE 14 bus and IEEE 30 bus cases respectively. The computational time required by each algorithm is also mentioned in each table. The losses presented in row 7 of table 1 and row 8 of table 2 are calculated from the generation values obtained from the best tradeoff solution.

Table 1. Results of CEED on IEEE 14 bus system

\begin{tabular}{|c|c|c|c|c|c|}
\hline Algorithm $\rightarrow$ & NSGA-II & SPEA2 & MOPSO & MODE & MMOCSO \\
\hline Gen1 in MW & 132.5353 & 139.2276 & 141.0835 & 142.3233 & 137.5120 \\
\hline Gen2 in MW & 52.4787 & 51.0889 & 50.9255 & 51.8410 & 50.6290 \\
\hline Gen3 in MW & 26.7157 & 26.0716 & 24.6853 & 24.7517 & 25.7137 \\
\hline Gen4 in MW & 30.7146 & 28.0029 & 27.1160 & 27.1009 & 29.1884 \\
\hline Gen5 in MW & 22.7507 & 21.0366 & 21.7250 & 19.6267 & 22.3171 \\
\hline Loss in MW & 6.3602 & 6.4276 & 6.5353 & 6.6436 & 6.1950 \\
\hline Run time in sec & 142.354601 & 166.064569 & 0.781151 & 5.1205 & 0.466674 \\
\hline Fuel Cost in \$/hour & 731.1801 & 726.5053 & 725.3175 & 727.8017 & 724.1148 \\
\hline Emission in lb/hour & 336.4998 & 342.9517 & 345.0662 & 347.7240 & 340.6991 \\
\hline
\end{tabular}

Table 2. Results of CEED of IEEE 30-Bus System

\begin{tabular}{|c|c|c|c|c|c|}
\hline Algorithm $\rightarrow$ & NSGA-II & SPEA2 & MOPSO & MODE & MMOCSO \\
\hline Gen1 in MW & 132.8562 & 140.8415 & 136.9922 & 135.1405 & 139.6455 \\
\hline Gen2 in MW & 51.5417 & 50.3428 & 51.3539 & 53.8810 & 51.8719 \\
\hline Gen3 in MW & 26.6750 & 25.0427 & 25.5302 & 25.3267 & 26.0681 \\
\hline Gen4 in MW & 31.0862 & 29.6649 & 31.0757 & 28.6958 & 29.9366 \\
\hline Gen5 in MW & 24.8491 & 22.5858 & 22.5543 & 24.6816 & 22.3233 \\
\hline Gen6 in MW & 23.6840 & 22.5781 & 23.4100 & 23.1221 & 21.1712 \\
\hline Loss in MW & 7.6166 & 7.6558 & 7.5163 & 7.4477 & 7.2922 \\
\hline Run time in sec & 222.308599 & 178.453432 & 0.80177 & 11.0430 & 0.441355 \\
\hline Fuel Cost (\$/hour) & 821.3096 & 815.1442 & 817.4411 & 818.7226 & 814.9821 \\
\hline Emission (lb/hour) & 379.2960 & 388.0709 & 384.1537 & 382.6765 & 387.7672 \\
\hline
\end{tabular}


Table 3. Performance Analysis of algorithms for IEEE 14 bus system

\begin{tabular}{|c|c|c|c|c|c|c|}
\hline \multicolumn{2}{|c|}{$\begin{array}{l}\text { Performance Index } \downarrow \\
\quad \text { Algorithm } \rightarrow\end{array}$} & $\begin{array}{l}\text { NSGA- } \\
\text { II }\end{array}$ & SPEA2 & MOPSO & MODE & MMOCSO \\
\hline \multirow[t]{4}{*}{ Spacing (S) } & Minimum & 0.4377 & 0.4136 & 0.6622 & 0.6042 & 0.4738 \\
\hline & Maximum & 0.9725 & 2.7420 & 0.6622 & 1.7268 & 0.4738 \\
\hline & Average & 0.6421 & 0.7623 & 0.6622 & 1.0120 & 0.4738 \\
\hline & $\begin{array}{c}\text { Std } \\
\text { Deviation }\end{array}$ & 0.1276 & 0.5437 & 0.0000 & 0.3058 & 0.0000 \\
\hline \multirow[t]{4}{*}{ Diversity $(\Delta)$} & Minimum & 0.4436 & 0.4480 & 0.5432 & 0.5228 & 0.4437 \\
\hline & Maximum & 0.8818 & 0.7552 & 0.5432 & 0.7589 & 0.4437 \\
\hline & Average & 0.6120 & 0.5325 & 0.5432 & 0.6532 & 0.4437 \\
\hline & $\begin{array}{c}\text { Std } \\
\text { Deviation }\end{array}$ & 0.0859 & 0.0640 & 0.0000 & 0.0584 & 0.0000 \\
\hline \multirow{4}{*}{$\begin{array}{c}\text { Spread Diversity } \\
\text { (SD) }\end{array}$} & Minimum & 65.5197 & 74.7557 & 93.3421 & 85.1830 & 94.1883 \\
\hline & Maximum & 97.8591 & 101.0925 & 93.3421 & 109.3091 & 94.1883 \\
\hline & Average & 78.4695 & 85.2477 & 93.3421 & 97.5195 & 94.1883 \\
\hline & $\begin{array}{c}\text { Std } \\
\text { Deviation }\end{array}$ & 8.9561 & 5.5098 & 0.0000 & 5.8995 & 0.0000 \\
\hline \multirow{4}{*}{$\begin{array}{c}\text { Mean Ideal } \\
\text { Distance (MID) }\end{array}$} & Minimum & 27.3435 & 31.6579 & 39.2568 & 32.0237 & 38.2848 \\
\hline & Maximum & 37.0736 & 37.3668 & 39.2568 & 39.8480 & 38.2848 \\
\hline & Average & 32.0023 & 34.7736 & 39.2568 & 35.0661 & 38.2848 \\
\hline & $\begin{array}{c}\text { Std } \\
\text { Deviation }\end{array}$ & 2.6608 & 1.4594 & 0.0000 & 1.8382 & 0.0000 \\
\hline
\end{tabular}

Table 4. Performance Analysis of Algorithms for IEEE 30-Bus System

\begin{tabular}{|c|c|c|c|c|c|c|}
\hline \multicolumn{2}{|c|}{$\begin{array}{l}\text { Performance Index } \downarrow \\
\text { Algorithm } \rightarrow\end{array}$} & NSGA-II & SPEA2 & MOPSO & MODE & MMOCSO \\
\hline \multirow[t]{4}{*}{ Spacing (S) } & Minimum & 0.4265 & 0.4694 & 1.0660 & 0.7574 & 0.5955 \\
\hline & Maximum & 0.6585 & 1.8672 & 1.0660 & 1.8367 & 0.5955 \\
\hline & Average & 0.5100 & 0.8577 & 1.0660 & 1.0955 & 0.5955 \\
\hline & $\begin{array}{c}\text { Std } \\
\text { Deviation }\end{array}$ & 0.0610 & 0.3500 & 0.0000 & 0.3343 & 0.0000 \\
\hline \multirow[t]{4}{*}{ Diversity $(\Delta)$} & Minimum & 0.3313 & 0.4151 & 0.5898 & 0.5587 & 0.4952 \\
\hline & Maximum & 0.6050 & 0.7012 & 0.5898 & 0.8315 & 0.4952 \\
\hline & Average & 0.4593 & 0.5292 & 0.5898 & 0.6629 & 0.4952 \\
\hline & $\begin{array}{c}\text { Std } \\
\text { Deviation } \\
\end{array}$ & 0.0771 & 0.0700 & 0.0000 & 0.0692 & 0.0000 \\
\hline \multirow{4}{*}{$\begin{array}{c}\text { Spread Diversity } \\
\text { (SD) }\end{array}$} & Minimum & 72.3583 & 101.2766 & 103.7742 & 96.2074 & 109.2431 \\
\hline & Maximum & 110.9874 & 118.8710 & 103.7742 & 123.4799 & 109.2431 \\
\hline & Average & 85.9714 & 106.6710 & 103.7742 & 110.7379 & 109.2431 \\
\hline & $\begin{array}{c}\text { Std } \\
\text { Deviation }\end{array}$ & 8.2858 & 4.1319 & 0.0000 & 6.6799 & 0.0000 \\
\hline \multirow{4}{*}{$\begin{array}{c}\text { Mean Ideal } \\
\text { Distance (MID) }\end{array}$} & Minimum & 33.4028 & 40.5709 & 47.6714 & 40.2759 & 43.6668 \\
\hline & Maximum & 42.4031 & 45.3736 & 47.6714 & 47.3750 & 43.6668 \\
\hline & Average & 37.7583 & 42.8149 & 47.6714 & 43.8900 & 43.6668 \\
\hline & $\begin{array}{c}\text { Std } \\
\text { Deviation } \\
\end{array}$ & 2.4397 & 1.3634 & 0.0000 & 1.7780 & 0.0000 \\
\hline
\end{tabular}

The algorithms are run for twenty-five times each, and the solutions obtained are analyzed. The quality of Pareto solutions has been assessed using the non-parametric performance metrics namely spacing, diversity, spread diversity and mean ideal distance. The minimum, maximum, 
average and standard deviation values of the metrics are presented in Table 3 and Table 4 for IEEE 14 bus and IEEE 30 bus test cases respectively. It shows that the proposed algorithm has the lowest average value of $\mathrm{S}$ and $\Delta$. It indicates that the solutions obtained are more uniformly distributed and exploit the search space better. The standard deviations of the metrics values are very low indicating that the search mechanism optimizes firmly without much deviations from the ideal non-dominating points. The average spread diversity value is larger than most of the competing algorithms except the MODE, indicating an acceptable performance in terms of spread of the Pareto front. Similarly, the value of MID is lower than that of MOPSO showing good dispersal of solutions from ideality.

All these algorithms under observation are based on random search techniques, so their performance cannot be judged by single observation. So, statistical performance testing is done by running the same algorithm for many times to ascertain their performance. A better value on any parameter is considered as a win and a worse one is said to be a loss. The two-tailed sign test is performed that shows the number of wins and losses for 25 runs of the proposed algorithm to test its superiority over the other four. The results are shown in Table 5 and Table 6 present a high degree of superiority i.e., $\alpha=0.05$ in favor of the proposed algorithm. It may be noted here that for 25 runs of the algorithms only 18 wins are required for a high degree of detected difference [28]. The performance of the same has been detected to be very good over the spacing and diversity metrics. It also outperforms three of the competing algorithms on the spread diversity metric and shows better results than MOPSO on mean ideal distance.

Table 5. Results of the sign test of MMOCSO on IEEE-14 bus system

\begin{tabular}{|c|c|c|c|c|c|}
\hline \multicolumn{2}{|c|}{ Winning Parameter $\downarrow$ Algorithm $\rightarrow$} & NSGA-II & SPEA2 & MOPSO & MODE \\
\hline \multirow{3}{*}{ Spacing (S) } & MMOCSO wins & 23 & 21 & 25 & 25 \\
\cline { 2 - 6 } & MMOCSO losses & 02 & 04 & 00 & 00 \\
\cline { 2 - 6 } & Detected difference & $\alpha=0.05$ & $\alpha=0.05$ & $\alpha=0.05$ & $\alpha=0.05$ \\
\hline Diversity ( $\Delta$ ) & MMOCSO wins & 24 & 25 & 25 & 25 \\
\cline { 2 - 6 } & MMOCSO losses & 01 & 00 & 00 & 00 \\
\cline { 2 - 6 } & Detected difference & $\alpha=0.05$ & $\alpha=0.05$ & $\alpha=0.05$ & $\alpha=0.05$ \\
\hline \multirow{3}{*}{$\begin{array}{c}\text { Spread Diversity } \\
\text { (SD) }\end{array}$} & MMOCSO wins & 24 & 24 & 25 & 07 \\
\cline { 2 - 6 } & MMOCSO losses & 01 & 01 & 00 & 18 \\
\cline { 2 - 6 } & Detected difference & $\alpha=0.05$ & $\alpha=0.05$ & $\alpha=0.05$ & -- \\
\hline Mean Ideal & MMOCSO wins & 00 & 00 & 25 & 01 \\
\cline { 2 - 6 } Distance (MID) & MMOCSO losses & 25 & 25 & 00 & 24 \\
\cline { 2 - 6 } & Detected difference & -- & -- & $\alpha=0.05$ & -- \\
\hline
\end{tabular}

Table 6. Results of the sign test of MMOCSO on IEEE-30 bus system

\begin{tabular}{|c|c|c|c|c|c|}
\hline \multicolumn{2}{|c|}{$\begin{array}{c}\text { Algorithm } \rightarrow \\
\text { Winning Parameter } \downarrow\end{array}$} & NSGA-II & SPEA2 & MOPSO & MODE \\
\hline \multirow{3}{*}{$\begin{array}{l}\text { Spacing } \\
\text { (S) }\end{array}$} & MMOCSO wins & 25 & 20 & 25 & 25 \\
\hline & MMOCSO losses & 00 & 05 & 00 & 00 \\
\hline & Detected difference & $\alpha=0.05$ & $\alpha=0.05$ & $\alpha=0.05$ & $\alpha=0.05$ \\
\hline \multirow{3}{*}{$\begin{array}{l}\text { Diversity } \\
(\Delta)\end{array}$} & MMOCSO wins & 25 & 17 & 25 & 25 \\
\hline & MMOCSO losses & 00 & 08 & 00 & 00 \\
\hline & Detected difference & $\alpha=0.05$ & $\alpha=0.1$ & $\alpha=0.05$ & $\alpha=0.05$ \\
\hline \multirow{3}{*}{$\begin{array}{c}\text { Spread } \\
\text { Diversity } \\
\text { (SD) }\end{array}$} & MMOCSO wins & 09 & 22 & 25 & 08 \\
\hline & MMOCSO losses & 16 & 03 & 00 & 17 \\
\hline & Detected difference & -- & $\alpha=0.05$ & $\alpha=0.05$ & -- \\
\hline \multirow{3}{*}{$\begin{array}{c}\text { Mean } \\
\text { Ideal } \\
\text { Distance } \\
\text { (MID) }\end{array}$} & MMOCSO wins & 16 & 06 & 25 & 16 \\
\hline & MMOCSO losses & 09 & 19 & 00 & 09 \\
\hline & Detected difference & -- & -- & $\alpha=0.05$ & -- \\
\hline
\end{tabular}


These results lead to the inference that, the proposed algorithm outperforms the competing algorithms under consideration with reference to spacing and diversity metrics without compromising much on the other two metrics taken. The results drawn in this paper are based on the tradeoff approach which allows a flexibility of assigning different importance to the objectives i.e., cost and emission through Pareto solutions; whereas some works [31] do not allow the user to choose such flexibility in decision.

\section{Conclusion and Future Scope}

The proposed modified multi-objective cat swarm optimization algorithm has been a successful tool for implementation of the combined economic emission dispatch problem. The solution obtained for two standard test cases i.e., IEEE 14 bus and 30 bus systems. A performance study is carried out on the proposed algorithm in comparison to two other competitive optimization algorithms. The Pareto optimal solutions obtained using the modified MOCSO algorithm show better diversity and improved exploitation of feasible search space. The proposed algorithm outperforms in terms of spacing, diversity and spread diversity metrics of the solution vectors obtained. It also has an edge over the competing algorithms with respect to the computational time required. The constraint violation i.e., the transmission losses are lower for the generation schedule obtained using the modified MOCSO algorithm. The fuel cost obtained for the latter is less and the corresponding generation schedule arrived leads to a lower transmission loss. Future improvement to the work can be done by the inclusion of a different local search technique based on different evolutionary algorithms. Critical analysis of the performance can be carried out by taking more realistic constraints such as power loss, prohibited operating zones etc. It may further improve the speed of convergence and exploration capability for multi-dimensional objective functions. The proposed algorithm may further be investigated on similar problems like voltage profile management, management of reactive power etc.

\section{Appendix}

The standard IEEE 14 bus and IEEE 30 bus test systems are taken as test cases for solution of the CEED problem of power systems. The parameters of these test cases have been adopted from standard sources. The coefficients of cost and emission functions used for solution of the problem are enumerated in tables A.1. and A.2. below.

Table A.1. IEEE 14 bus system cost and emission coefficients

\begin{tabular}{|c|c|c|c|c|c|}
\hline Gen no & 1 & 2 & 3 & 4 & 5 \\
\hline Max MW & 250 & 140 & 100 & 120 & 45 \\
\hline Min MW & 10 & 20 & 15 & 10 & 10 \\
\hline$\gamma$ & 0.0126 & 0.02 & 0.027 & 0.0291 & 0.029 \\
\hline$\beta$ & -0.9 & -0.1 & -0.01 & -0.005 & -0.004 \\
\hline$\alpha$ & 22.983 & 25.313 & 25.505 & 24.9 & 24.7 \\
\hline $\mathrm{a}$ & 0.00375 & 0.0175 & 0.0625 & 0.00834 & 0.025 \\
\hline $\mathrm{b}$ & 2.0 & 1.75 & 1.0 & 3.25 & 3.0 \\
\hline $\mathrm{c}$ & 0 & 0 & 0 & 0 & 0 \\
\hline
\end{tabular}

B Coefficients of IEEE 14 bus system used are

$$
\begin{aligned}
& B=\left[\begin{array}{rrrrrr}
0.0208 & 0.0090 & -0.0021 & 0.0024 & 0.0006 \\
0.0090 & 0.0168 & -0.0028 & 0.0035 & 0.0000 \\
-0.0021 & -0.0028 & 0.0207 & -0.0152 & -0.0179 \\
0.0024 & 0.0035 & -0.0152 & 0.0763 & -0.0103 \\
0.0006 & 0.0000 & -0.0179 & -0.0103 & 0.0476
\end{array}\right] \\
& B 0=\left[\begin{array}{lllll}
-0.0001 & 0.0023 & -0.0012 & 0.0027 & 0.0011
\end{array}\right] \\
& B 00=3.1826 \times 10^{-4}
\end{aligned}
$$


Table A.2. IEEE 30 bus system cost and emission coefficients

\begin{tabular}{|c|c|c|c|c|c|c|}
\hline Gen no & 1 & 2 & 3 & 4 & 5 & 6 \\
\hline $\begin{array}{c}\text { Max } \\
\text { MW }\end{array}$ & 200 & 80 & 50 & 35 & 30 & 40 \\
\hline $\begin{array}{c}\text { Min } \\
\text { MW }\end{array}$ & 50 & 20 & 15 & 10 & 10 & 12 \\
\hline$\gamma$ & 0.0126 & 0.02 & 0.027 & 0.0291 & 0.029 & 0.0271 \\
\hline$\beta$ & -0.9 & -0.1 & -0.01 & -0.005 & -0.0004 & -0.0055 \\
\hline$\alpha$ & 22.983 & 25.313 & 25.505 & 24.9 & 24.7 & 25.3 \\
\hline $\mathrm{a}$ & 0.00375 & 0.0175 & 0.0625 & 0.00834 & 0.025 & 0.025 \\
\hline $\mathrm{b}$ & 2.0 & 1.7 & 1.0 & 3.25 & 3.0 & 3.0 \\
\hline $\mathrm{c}$ & 0 & 0 & 0 & 0 & 0 & 0 \\
\hline
\end{tabular}

B Coefficients of IEEE 30 bus system used are

$$
\begin{aligned}
& B=\left[\begin{array}{lllllll}
0.0218 & 0.0103 & 0.0010 & -0.0025 & 0.0007 & 0.0033 \\
0.0103 & 0.0233 & 0.0001 & -0.0043 & 0.0009 & 0.0032 \\
0.0010 & 0.0001 & 0.0525 & -0.0380 & -0.0111 & -0.0066 \\
-0.0025 & -0.0043 & -0.0380 & -0.1011 & 0.0132 & 0.0045 \\
0.0007 & 0.0009 & -0.0111 & 0.0132 & 0.0163 & -0.0001 \\
0.0033 & 0.0032 & -0.0066 & 0.0045 & -0.0001 & 0.0270
\end{array}\right] \\
& B 0=\left[\begin{array}{llllll}
-0.0002 & 0.0029 & -0.0033 & 0.0035 & 0.00016 & 0.0048
\end{array}\right] \\
& B 00=0.0025
\end{aligned}
$$

\section{References}

[1]. H. Sadaat, Power System Analysis. McGraw-Hill Publishing Company Limited, 2009.

[2]. A. J. Wood and B. F. Wollenberg, Power Generation, Operation and Control. John Wiley and Sons, Inc., 1998.

[3]. A. Farag, Al-Baiyat and S. T. C. Cheng, "Economic load dispatch multiobjective optimization procedures using linear programming techniques", IEEE Transaction on Power System, pp. 731-738, 1995.

[4]. L. F. Wang and C. Singh, "Multi-objective stochastic power dispatch through a modified particle swarm optimization algorithm", Special Session on Applications of Swarm Intelligence to Power Systems, Proceedings of IEEE Swarm Intelligence Symposium, pp. 127-135, 2006.

[5]. M. A. Abido, "Environmental/Economic Power Dispatch Using Multi-Objective Evolutionary Algorithms", IEEE Transaction on Power System, Vol. 18, Issue 4, pp. 15291537, 2003.

[6]. C. L. Chiang, J. H. Liaw and C. T. Su, "New approach with a genetic algorithm framework to multi-objective generation dispatch problems", European Transactions on Electrical Power, Vol. 15, pp. 381-395, 2005.

[7]. K. Deb, A. Pratap, S. Agarwal, and T. Meyariyan, "A Fast and Elitist Multiobjective Genetic Algorithm: NSGA-II", IEEE Transactions on Evolutionary Computation, Vol. 6, Issue 2, pp. 182-197, 2002.

[8]. R. T. F. A. King, H. C. S. Rughooputh and K. Deb, "Evolutionary Multi-Objective Environmental/Economic Dispatch: Stochastic vs. Deterministic Approaches”, Kanpur GA Lab Report, Number 019, 2004.

[9]. Y. S. Brar, J. S. Dhillon, and D. P. Kothari, "Multi-objective Load Dispatch Based on Genetic-Fuzzy Technique", IEEE Conference on PSCE, pp. 931-937, 2006.

[10]. R. Muthuswamy, M. Krishnan, K. Subramanian, and B. Subramanian, "Environmental and economic power dispatch of thermal generators using modified NSGA-II algorithm", International Transactions on Electrical Energy Systems, Vol. 25, pp. 1552-1569, 2015. 
[11]. K. Nayak, R. Krishnanand, B. K. Panigrahi, and P. K. Rout, “Application of Artificial Bee Colony to Economic Load Dispatch Problem with Ramp Rate Limits and Prohibited Operating Zones", IEEE World Congress on Nature \& Biologically Inspired Computing, pp. 1237-1242, 2009.

[12]. R. H. Liang, C. Y. Wu, Y. T. Chen and W. T. Tseng, "Multi-objective dynamic optimal power flow using improved artificial bee colony algorithm based on Pareto optimization", International Transactions on Electrical Energy Systems, Vol. 26, pp. 692-712, 2016.

[13]. H. Mori, and K. Okawa, "Advanced MOEPSO-based Multi-Objective Environmental Economic Load Dispatching”, IEEE Conference, doi: 10.1109/PES.2010.5590209, 2010.

[14]. B. Hadji, B. Mahdad, K. Srairi, and N. Mancer, "Multi-objective PSO-TVAC for Environmental Economic Dispatch Problem", Intl. Conf. on Technologies and Materials for Renewable Energy and Sustainability TMREES15, Energy Procedia, Elsevier, pp. 102$111,2015$.

[15]. R. Storn and K. Price, "Differential Evolution - A Simple and Efficient Heuristic for Global Optimization over Continuous Spaces", Journal of Global Optimization, Vol. 11, Issue 4, pp. 341-359,1997.

[16]. G. R. Meza, X. Blasco, J. Sanchis and M. Martinez, "Multiobjective optimization algorithm for solving constrained single objective problems", IEEE Conference, doi: 10.1109/CEC.2010.5586408, 2010.

[17]. Y. Di, M. Fei, L. Wang and W. Wu, "Multi-objective optimization for economic emission dispatch using an improved multi-objective binary differential evolution algorithm", The 6th International Conference on Applied Energy - ICAE, Energy Procedia, Elsevier, doi: 10.1016/j.egypro.2014.12.065, 2014.

[18]. M. Kuo, S. Lu and M. Tsou, "Considering Carbon Emissions in Economic Dispatch Planning for Isolated Power Systsems-A Case Study of the Taiwan Power System", IEEE Transactions on Industry Applications, doi: 10.1109/TIA.2017.2771338, 2017

[19]. E. Arriagada, E. Lopez, M. Lopez, G. Lefranc, R. Lopez and M. Poloujadoff, “A Probabilistic Economic/ $\mathrm{CO}_{2 \text { eq }}$ Emissions Dispatch Model: Real Applications", IEEE Latin America Transactions, Vol. 16. No. 9, pp. 2362-2369, 2018.

[20]. A. A. Elsakaan, R. A. El-Seheimy, S. S. Kaddah and M. I. Elsaid, "Optimal Economic Emission Power Schedulingof RERs in MGs with Uncertainty", IET Generation, Transmission and Distribution, Vol. 14, Iss. 1, pp. 37-52, 2019.

[21]. R. Dong and S. Wang, "New Optimization Algorithm Inspired by Kernel Tricks for the Economic Emission Dispatch Problem with Valve Point", IEEE Access, Vol. 8, pp. 16584$16594,2020$.

[22]. J. Sun, J. Deng and Y. Li, "Indicator and Crowding Distance based Evolutionary Algorithm for Combined Heat and Power Economic Emission Dispatch", Applied Soft Computing Journal, Elsevier, 90 (2020) 106158, pp. 1-15, 2020.

[23]. E. Zitler, M. Laumanns and L. Thiele, "SPEA2: Improving the Strength Pareto Evolutionary Algorithm", Evolutionary Methods for Design Optimization and Control with Applications to Industrial Problems, pp. 1-19, 2001.

[24]. R. C. Eberhart and J. Kennedy, "A New Optimizer using Particle Swarm Theory", Sixth International Symposium on Micro-machine and Human Science, pp. 39-43, 1995.

[25]. V. Hosseinnezhad, M. Rafiee, Md. Ahmedian and Md. Taghi Ameli, "Species-based Quantum Particle Swarm Optimization for economic load dispatch", Electrical Power and Energy Systems, Elsevier, Vol. 63, pp. 311-332, 2014.

[26]. S. K. Mishra and S. K. Mishra, "Solution of Constrained Economic Emission Dispatch Problem Using Multi-Objective Particle Swarm Optimisation”, International Journal of Control Theory and Applications, Vol. 9, Issue 39, pp. 63-70, 2016.

[27]. P. M. Pradhan, G. Panda, "Solving Multiobjective Problems using Cat Swarm Optimization", Expert Systems with Applications, Elsevier, Vol. 39, pp. 2956-2964, 2012. 
[28]. S. K. Mishra, G. Panda and R. A. Majhi, "Comparative Performance Assessment of a Set of Multi-Objective Algorithms for Constrained Portfolio Assets Selection", Swarm and Evolutionary Computing, Elsevier, Vol. 16, pp. 38-51, 2014.

[29]. M. Andervazh, S. Javadi, "Emission-economic dispatch of thermal power generation units in the presence of hybrid electric vehicles and correlated wind power plants", IET Generation, Transmission and Distribution, Vol. 11, Issue 9, pp. 2232-2243, 2017.

[30]. S. P. Karthikeyan, K. Palanisamy, C. Rani, I. J. Ragland and D. P. Kothari, "Security Constrained Unit Commitment Problem with Operational Power Flow and Environmental Constraints", WSEAS Transactions on Power Systems, Vol. 4, Issue 2, pp. 53-66, 2009.

[31]. B. F. Zohra, A. K. Lahouari and R. Mostefa, "An Improved Harmony Search Algorithm for solved the Combined Heat and Power Economic Dispatch", International Journal on Electrical Engineering and Informatics, Vol. 11, No. 2, pp. 440-450, 2019.

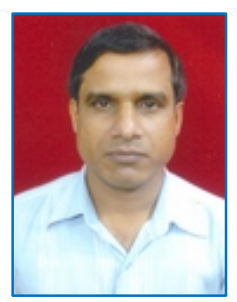

Sarat Kumar Mishra is a $\mathrm{PhD}$ scholar in the Electrical and Electronics Engineering Department of Birla Institute of Technology, Mesra, Ranchi, India. He received his M.E. degree in Electrical Engineering from National Institute of Technology, Rourkela in 1998. He completed his B.E. degree in Electrical Engineering from Indira Gandhi Institute of Technology, Sarang under Utkal University, Odisha, India. He is currently working as Assistant Professor in the Electrical and Electronics Engineering Department of Padmanava College of Engineering, Rourkela, India. His research interest includes power system operation and planning. mishra.sarat@gmail.com

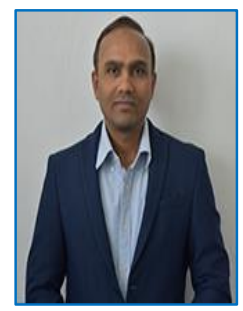

Sudhansu Kumar Mishra is Assistant Professor in the Electrical and Electronics Engineering Department of Birla Institute of Technology, Mesra, Ranchi, India. His research areas include signal processing, control system, biomedical image processing, computational finance, soft and evolutionary computing. sudhansu.nit@gmail.com 